\title{
Downregulation of microRNA-26a is associated with metastatic potential and the poor prognosis of osteosarcoma patients
}

\author{
QI-CHUN SONG ${ }^{1}$, ZHI-BIN SHI $^{1}$, YONG-TAO ZHANG ${ }^{1}$, LE JI $^{1}$, KUN-ZHENG WANG $^{1}$, \\ DA-PENG DUAN ${ }^{2}$ and XIAO-QIAN DANG ${ }^{1}$ \\ ${ }^{1}$ Department of Orthopedics, The Second Affiliated Hospital, Xi'an Jiaotong University, Xi'an, Shaanxi 710004; \\ ${ }^{2}$ Department of Orthopedics, Shaanxi Provincial People's Hospital, Xi'an, Shaanxi 710068, P.R. China
}

Received November 16, 2013; Accepted December 30, 2013

DOI: $10.3892 /$ or.2014.2989

\begin{abstract}
Accumulating evidence indicates that microRNAs are involved in multiple processes in cancer development and progression. microRNA-26a (miR-26a) has been identified as a tumor suppressor and its downregulation is associated with poor prognosis in several types of human cancer. However, the specific function of miR-26a in osteosarcoma remains unclear. In the present study, we found that the expression of miR-26a in osteosarcoma tissues and cell lines was much lower than that in the normal controls, respectively. In addition, downregulation of miR-26a more frequently occurred in osteosarcoma specimens with adverse clinical stage and with the presence of distant metastasis. Moreover, multivariate survival analyses demonstrated that loss of miR-26a is an independent prognostic factor for both disease-free and overall survival in osteosarcoma. In addition, restoration of miR-26a expression inhibited the invasion and migration in osteosarcoma cells, and miR-26a directly inhibited enhancer of zeste homolog 2 (EZH2) expression by targeting its 3'-UTR. Moreover, EZH2 was upregulated and inversely correlated with miR-26a expression in the osteosarcoma tissues. Thus, for the first time, we provide convincing evidence that downregulation of miR-26a is associated with tumor aggressiveness and tumor metastasis, and miR-26a inhibits cell migration and invasion by targeting the EZH2 gene in osteosarcoma. Thus, miR-26a is an independent prognostic marker for osteosarcoma patients.
\end{abstract}

Correspondence to: Professor Xiao-Qian Dang, Department of Orthopedics, The Second Affiliated Hospital, Xi'an Jiaotong University, 157 Xiwu Road, Xi'an, Shaanxi 710004, P.R. China E-mail: xqdang@163.com

Abbreviations: miR-26a, miRNA-26a; EZH2, enhancer of zeste homolog 2; PcG, polycomb group; WT, wild-type; MT, mutant; 3'-UTR, 3'-untranslated region; qRT-PCR, quantitative real-time polymerase chain reaction

Key words: miR-26a, osteosarcoma, metastasis, prognosis, EZH2

\section{Introduction}

Osteosarcoma, mainly arising from the metaphysis of the long bones, is the most common primary malignancy of bone in adolescents and young adults, with an estimated worldwide yearly incidence rate of 4 million $(1,2)$. Despite current therapeutic strategies combining adjuvant chemotherapy, surgery and sometimes radiotherapy, the prognosis of osteosarcoma patients remains poor, since $\sim 80 \%$ of patients eventually develop recurrent metastatic osteosarcoma following surgical treatment (3), and the 5-year survival rate of these patients is only $50-60 \%$ (4). Although recent developments in molecular biology have provided insight into the molecular mechanisms of osteosarcoma, the fundamental molecular mechanisms underlying metastasis in osteosarcoma have not been fully elucidated. Therefore, it is essential to identify metastasisassociated molecules as effective drug targets and to enhance the understanding of the mechanisms underlying the metastasis of osteosarcoma.

MicroRNAs (miRNAs) are small non-coding RNAs 18-25 nucleotides in length, transcribed from non-proteincoding genes or introns, which regulate gene expression through repressing translation and cleaving their target mRNAs by binding to complementary sites in their 3'-untranslated region (3'-UTR). It has been demonstrated that aberrant expression of miRNAs cause them to function as tumor suppressors or oncogenes according to the roles of their target genes $(5,6)$. Particularly, miRNAs can regulate various cellular processes of tumor cells, including differentiation, progression, apoptosis, proliferation, migration and invasion (7). To date, several human miRNAs such as miR-335, miR-145 and miR-128 have been shown to be dysregulated in osteosarcoma (7-9), and contribute to the development and progression of osteosarcoma.

Emerging data reveal that microRNA-26a (miR-26a) is downregulated and may serve as a potential tumor suppressor in several distinct cancer types including nasopharyngeal carcinoma, breast cancer, thyroid anaplastic carcinomas and hepatocellular carcinoma (5,10-14). Importantly, miR-26a was found to be highly expressed in lymph node metastatic tumors as compared with primary tumors and enhanced lung cancer cell migration and invasion (15). miR-26a can suppress cell differentiation, migration and invasion by targeting a number 
of important genes such as SMAD1, MTDH, CDK6, CCNE1, CCNE2 CCND2, PTEN, PB1, MAP3K2 and enhancer of zeste homolog 2 (EZH2) (13,15-20). However, the roles of miR-26a and its regulated targets in osteosarcoma have not yet been clarified.

In the present study, we investigated the potential function of miR-26a in the development and progression of osteosarcoma. We found that miR-26a expression was downregulated in the majority of osteosarcoma tissues, and downregulation of miR-26a was significantly associated with tumor recurrence, metastasis and poor prognosis in osteosarcoma patients. Moreover, in vitro assays showed that miR-26a was significantly associated with suppressed tumor invasion and metastasis of osteosarcoma cells by targeting its direct target EZH2. To the best of our knowledge, this is the first study to examine the expression and role of miR-26a in osteosarcoma prognosis and metastasis.

\section{Materials and methods}

Patients and tissue samples. The present study was approved by the Research Ethics Committee of Xi'an Jiaotong University. Written informed consent was obtained from all of the patients. All specimens were handled and made anonymous according to the ethical and legal standards. A total of 144 patients were enrolled in this study. Patients received curative resection for osteosarcoma at the Second Affiliated Hospital, Xi'an Jiaotong University (Xi'an, China) between 2001 and 2008. No patients had received blood transfusion, radiotherapy or chemotherapy prior to surgery. The clinicopathological information of these patients is documented in Table I. The follow-up information of all participants was updated every 3 months by telephone. The overall survival was defined as the time elapsed from surgery to death. Information regarding the death of patients was ascertained from their family. Patients were followed up after surgical treatment until July 2012, with a median follow-up of 83 months (range, 12-139 months). During the follow-up period, 65 patients (45.1\%) died of disease. Distant metastases developed in 37 patients at a mean of 15.1 months (range, 4-39 months) after initial diagnosis. The median overall and disease-free survival of patients was 43 and 37 months, respectively.

Quantitative reverse transcriptase PCR ( $q R T-P C R)$ assay. The expression of miR-26a in the osteosarcoma and corresponding non-cancer tissues was determined by qRT-PCR assay. Briefly, total RNA was extracted from the tissues using TRIzol reagent (Invitrogen, Carlsbad, CA, USA) according to the manufacturer's protocol. miRNA expression levels were then quantitated using the TaqMan miRNA real-time RT-PCR kit (Applied Biosystems) according to the manufacturer's protocol. Data were analyzed using 7500 software v.2.0.1 (Applied Biosystems), with the automatic Ct setting for adapting baseline and threshold for $\mathrm{Ct}$ determination. The universal small nuclear RNA U6 (RNU6B) was used as an endogenous control for miRNAs. Each sample was examined in triplicate, and the amounts of PCR products produced were non-neoplasticized to RNU6B.

Cell culture. Human osteosarcoma cell lines MG-63 and U20S were obtained from the Cell Bank of the Chinese Academy
Table I. Association of miR-26a expression with the clinicopathological features of the osteosarcoma cases.

\begin{tabular}{|c|c|c|c|c|}
\hline \multirow[b]{2}{*}{$\begin{array}{l}\text { Clinicopathological } \\
\text { features }\end{array}$} & \multirow[b]{2}{*}{$\begin{array}{l}\text { No. of } \\
\text { cases }\end{array}$} & \multicolumn{2}{|c|}{ miR-26a expression } & \multirow[b]{2}{*}{ P-value } \\
\hline & & $\begin{array}{l}\text { High } \\
\mathrm{n}(\%)\end{array}$ & $\begin{array}{l}\text { Low } \\
\mathrm{n}(\%)\end{array}$ & \\
\hline Age (years) & & & & 0.865 \\
\hline$<55$ & 62 & $34(54.8)$ & $28(45.2)$ & \\
\hline$\geq 55$ & 82 & $47(57.3)$ & $35(42.7)$ & \\
\hline Gender & & & & 0.738 \\
\hline Male & 76 & 44 (57.9) & $32(42.1)$ & \\
\hline Female & 68 & $37(54.4)$ & $31(45.6)$ & \\
\hline Tumor size $(\mathrm{cm})$ & & & & 0.311 \\
\hline$>8$ & 79 & $41(51.9)$ & $38(48.1)$ & \\
\hline$\leq 8$ & 65 & $40(61.5)$ & $25(38.5)$ & \\
\hline Anatomic location & & & & 0.611 \\
\hline Femur & 78 & $44(56.4)$ & $34(43.6)$ & \\
\hline Tibia & 42 & $21(50.0)$ & $21(50.0)$ & \\
\hline Humeral bone & 17 & $11(64.7)$ & $6(35.3)$ & \\
\hline Other & 7 & $5(71.4)$ & $2(28.6)$ & \\
\hline Clinical stage & & & & $0.043^{\mathrm{a}}$ \\
\hline IIA & 62 & $41(66.1)$ & $21(33.9)$ & \\
\hline IIB/III & 82 & $40(48.8)$ & $42(51.2)$ & \\
\hline Metastasis & & & & $0.004^{\mathrm{a}}$ \\
\hline Present & 37 & $13(35.1)$ & $24(64.9)$ & \\
\hline Absent & 107 & $68(63.6)$ & $39(36.4)$ & \\
\hline $\begin{array}{l}\text { Response to } \\
\text { chemotherapy }\end{array}$ & & & & $0.044^{\mathrm{a}}$ \\
\hline Favorable & 69 & $45(65.2)$ & $24(34.8)$ & \\
\hline Poor & 75 & $36(48.0)$ & $39(52.0)$ & \\
\hline
\end{tabular}

${ }^{\mathrm{a}} \mathrm{P} \leq 0.05$

of Sciences (Shanghai, China), where they were characterized by mycoplasma detection, DNA-fingerprinting, isozyme detection and cell vitality detection. They were cultured in Dulbecco's modified Eagle's medium (DMEM) (Invitrogen) supplemented with $10 \%$ fetal bovine serum (FBS) (HyClone, Logan, UT, USA) and cultured in a humidified incubator at $37^{\circ} \mathrm{C}$ in $5 \% \mathrm{CO}_{2}$.

In vitro migration and invasion assays. Cell migration and invasion capacities were assessed in vitro using Transwell migration assays (Millipore, Billerica, MA, USA). The osteosarcoma cells were transfected with miR-26a mimics, inhibitor or scramble for $48 \mathrm{~h}$ and suspended in DMEM with $10 \mathrm{~g} / 1 \mathrm{BSA}$ at a density of 50 cells $/ \mathrm{ml}$. Then, cell suspensions $(200 \mu \mathrm{l})$ were seeded into the upper chamber with a porous membrane coated with (for the Transwell invasion assay) or without (for the migration assay) Matrigel (BD Biosciences, San Diego, CA, USA). To attract the cells, $500 \mu 1$ of DMEM with $10 \%$ serum was added to the bottom chamber. After allowing the cells to migrate for $24 \mathrm{~h}$ or to invade for $48 \mathrm{~h}$, the penetrated 
A
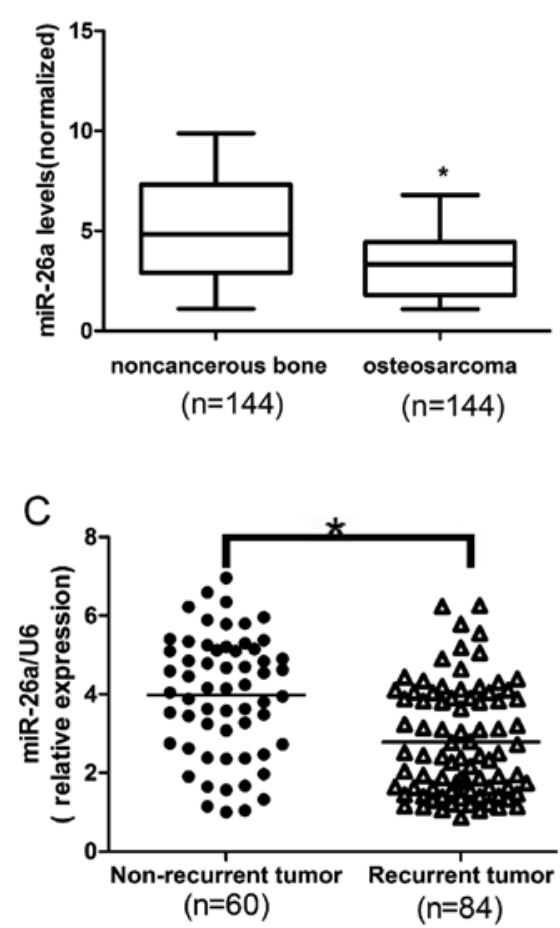

B

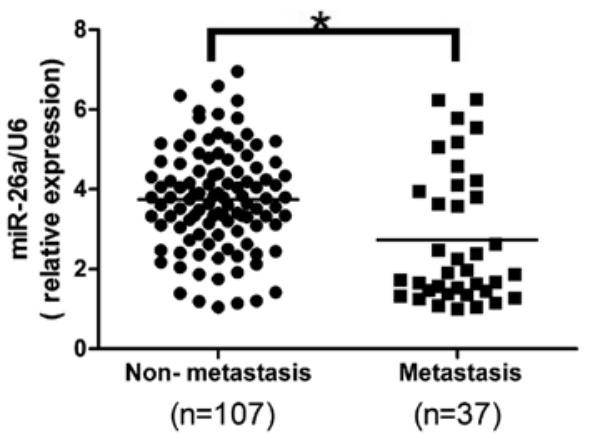

D

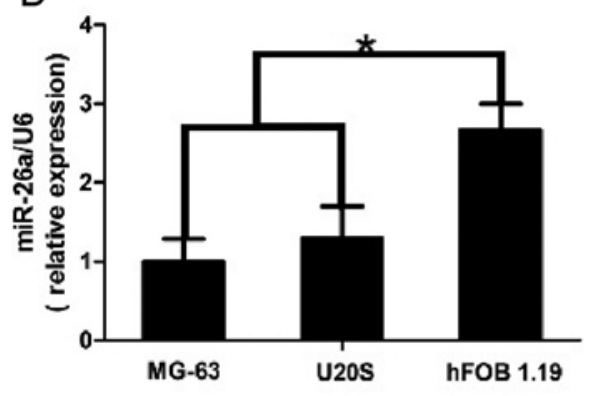

Figure 1. Expression levels of miR-26a in osteosarcoma tissues and cell lines. (A) The relative expression of miR-26a in 144 paired human osteosarcoma tissues and their corresponding normal samples. (B) Comparison of the expression of miR-26a between metastatic and non-metastatic osteosarcoma tissues. (C) Comparison of the expression of miR-26a between tumor tissues from the recurrent and non-recurrent group. (D) The relative expression levels of miR-26a in osteosarcoma cell lines and human osteoblast cell line hFOB 1.19. Expression levels of miR-26a were determined by qRT-PCR and normalized against an endogenous control U6 RNA; ${ }^{\mathrm{P}}<0.05$.

cells on the filters were fixed in dried methanol and stained in $4 \mathrm{~g} / \mathrm{l}$ crystal violet. The numbers of migrated or invasive cells were determined from five random fields using a microscope (Olympus) at x10 magnification.

Oligonucleotide transfection. miR-26a mimics and inhibitor were chemically synthesized by Shanghai GenePharma (Shanghai, China). When the cells achieved $80 \%$ confluence, miR-26a mimics or the inhibitor was transfected into the osteosarcoma cells with Lipofectamine 2000 (Invitrogen) according to the manufacturer's instructions. Cells were also transfected with a scramble oligonucleotide as a negative control (NC). The expression level of miR-26a in the transfected osteosarcoma cells was determined by qRT-PCR.

Luciferase reporter assay. Osteosarcoma cells were seeded into a 96-well plate at $60 \%$ confluence. After $24 \mathrm{~h}$, cells were transfected with $120 \mathrm{ng}$ of miR-26a expression vector or the negative control. Cells were transfected with $30 \mathrm{ng}$ of wildtype (WT) or mutant (MT) 3'-UTR of EZH2 mRNA. Cells were collected $48 \mathrm{~h}$ after transfection, and the luciferase activity was measured using a dual-luciferase reporter assay system according to the manufacturer's protocol (Promega).

Western blotting. Cells were harvested in lysis buffer $(50 \mathrm{mM}$ $\mathrm{NaCl}, 50 \mathrm{mM}$ EDTA, 1\% Triton X-100) containing protease inhibitor cocktail (Roche, Indianapolis, IN, USA). The cell lysates $(30 \mu \mathrm{g})$ were separated using $10 \%$ SDS-PAGE gels and then transferred onto nitrocellulose membranes (Millipore, Bedford, MA, USA). The membranes were blocked with
$5 \%$ non-fat milk diluted in PBS for $2 \mathrm{~h}$ at room temperature before the addition of the appropriate primary antibody. The antibodies used in the present study included anti-EZH2 $(1: 1000 ;$ ab3748) and anti- $\beta$-actin $(1: 1,000$; ab14128) (both from Abcam). The membranes were then washed with PBS containing $0.05 \%$ Tween and incubated with the appropriate HRP-conjugated secondary antibody (1:10,000; Abcam) for $1 \mathrm{~h}$ at room temperature. The bands were visualized using a chemiluminescence reagent (New England Nuclear, Boston, MA, USA).

Statistical analysis. Statistical analysis was performed using IBM SPSS statistical software (version 20.0). Survival curves were estimated using the Kaplan-Meier method, and distributions were evaluated by the log-rank test. Cox proportional hazard models of factors related to survival were used to calculate risk ratios (RRs) and identify the factors that affected survival. The differences in characteristics between two groups were examined by the $\chi^{2}$ test and Fisher's exact test. All P-values were determined from 2-sided tests, and statistical significance was based on a P-value of 0.05 .

\section{Results}

Downregulation of miR-26a is associated with metastasis and recurrence of osteosarcoma. Expression of miR-26a was analyzed in 144 pairs of osteosarcoma and corresponding non-cancerous bone tissues by quantitative real-time polymerase chain reaction (qRT-PCR) and normalized against an endogenous control (U6 RNA). As shown in Fig. 1A, the 
expression level of miR-26a in the osteosarcoma tissues was found to be distinctly downregulated when compared to that in the non-cancerous bone tissues. Furthermore, in comparison to the non-metastatic osteosarcoma tissues, the miR-26a levels were significantly lower in the metastatic osteosarcoma tissues (Fig. 1B). Moreover, miR-26a levels were decreased in the tumor tissues obtained from the patients who suffered osteosarcoma recurrence in comparison with patients who did not have tumor recurrence (Fig. 1C). These data indicate that significant downregulation of miR-26a expression occurred in osteosarcoma and was correlated with osteosarcoma relapse and metastasis. To further evaluate the association of miR-26a with osteosarcoma metastasis, we analyzed miR-26a levels in human osteosarcoma cell lines, MG-63 and U20S, and human osteoblast cell line hFOB 1.19. Similarly, we found that expression of miR-26a was much lower in the osteosarcoma cell lines than that in the osteoblast cell line (Fig. 1D). Collectively, the above findings suggest that loss of miR-26a expression is correlated with increased metastatic potential of osteosarcoma cells.

Downregulation of miR-26a is associated with advanced clinicopathological features of osteosarcoma. To determine the clinical significance of miR-26a in osteosarcoma, we analyzed the association of miR-26a expression with various clinicopathological parameters of osteosarcoma tissues. The median miR-26a expression level in all 144 patients with osteosarcoma was 3.37. The patients were divided into two groups according to their expression levels of miR-26a, using the median level as a cut off: the high miR-26a expression group $(n=81)$ and the low miR-26a expression group $(n=63)$. As shown in Table I, miR-26a was significantly downregulated in osteosarcoma patients with advanced clinical stage $(\mathrm{P}=0.043)$ and positive distant metastasis $(\mathrm{P}=0.004)$. Taking into consideration the relationship between miR-26a expression and the response to chemotherapy, we found that patients with low miR-26a had a poorer response to chemotherapy than those with high miR-26a expression $(\mathrm{P}=0.044)$, whereas, miR-26a expression was not significantly correlated with gender, age, tumor size, anatomic location or alkaline phosphatase level.

Downregulation of miR-26a is associated with poor prognosis in patients with osteosarcoma. To determine the prognostic value of miR-26a expression in osteosarcoma, we analyzed the relationship between the miR-26a expression and clinical outcome. The relationship between miR-26a expression and overall survival or disease-free survival was investigated using Kaplan-Meier analysis and log-rank test. A statistically significant difference in overall survival and disease-free survival was found between the high miR-26a expression group and the low miR-26a expression group (Fig. 2A and B; log-rank test, $\mathrm{P}=0.0009$ and $\mathrm{P}<0.0001$, respectively). The patients with low miR-26a expression tended to have a shorter overall and disease-free survival time when compared to patients with high miR-26a expression. In the multivariate analysis, we found that low miR-26a expression was associated with a decreased overall and disease-free survival. The adjusted risk ration (RR) was 5.724 (95\% CI, 1.008-10.991; $\mathrm{P}=0.007)$ and 3.972 (95\% CI, 1.191-9.871; $\mathrm{P}=0.014$ ), respectively, indicating that the expression of miR-26a may be a prognostic factor for
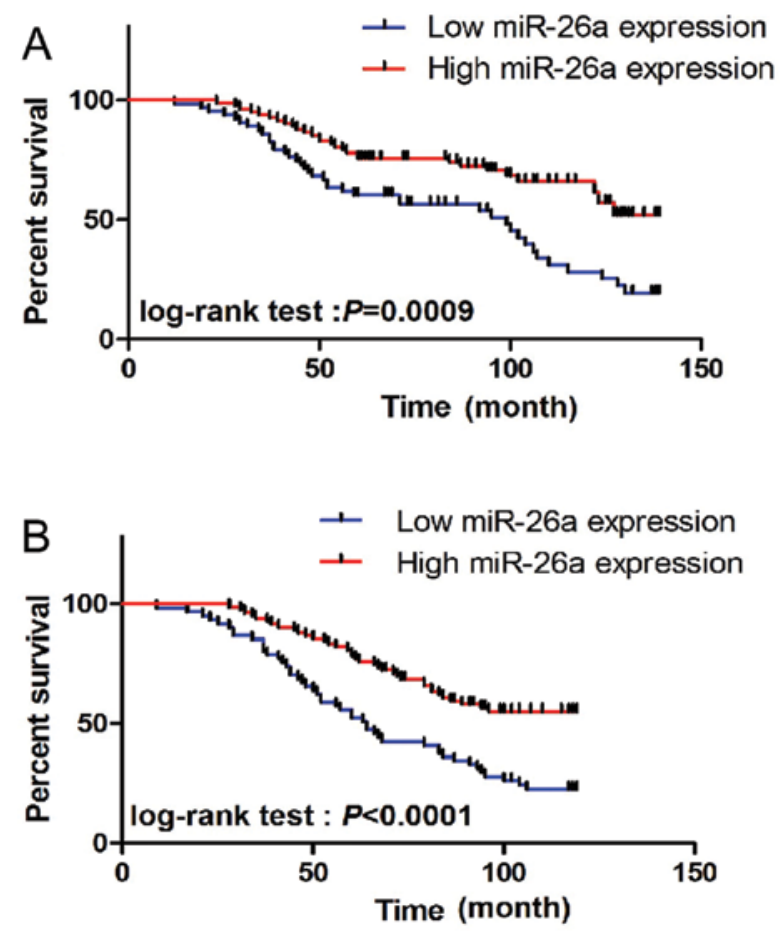

Figure 2. Relationship between miR-26a expression and overall and diseasefree survival time. (A) Overall and (B) disease-free survival curves for two groups of osteosarcoma patients with low and high expression of miR-26a.

overall and disease-free survival independent of these adjusted clinicopathologic characteristics (Table II).

$m i R-26$ a reduces the migration and invasion of osteosarcoma cells. As patients with recurrent metastatic osteosarcoma present with a poor outcome and based on our previous results indicating that miR-26a is significantly associated with disease-free survival and prognosis, we aimed to ascertain whether miR-26a affects the cell migration and invasion of osteosarcoma cells. To confirm the effect of miR-26a on cell migration and invasion, MG-63 and U2OS cells were transiently transfected with miR-26a mimics or the miR-26a inhibitor, respectively. As expected, transfection of miR-26a mimics or the miR-26a inhibitor resulted in an increase and decrease, respectively, in miR-26a expression when compared to the negative control (Fig. 3A and B). Moreover, the results of the migration and invasion assays revealed that miR-26a restoration resulted in reduced migration and invasion of MGG-63 and U2OS cells when compare to the control, whereas, antimiR-26a promoted cell migration and invasion (Fig. 3C-F). These results indicate that miR-26a functions as a tumor suppressor miRNA and contributes to the inhibition of migration and invasion of osteosarcoma cells.

EZH2 is a direct target of miR-26a in osteosarcoma cells. To elucidate the mechanism of inhibition involved in the migration and invasion in human osteosarcoma by miR-26a, we searched for candidate genes for miR-26a using publicly available databases, including TargetScan, PicTar and miRanda. EZH2 was selected for further experimental validation, since the complementary sequence of miR-26a was identified in the 3'-UTR of EZH2 mRNA by miRanda analysis (Fig. 4A). To 
Table II. Multivariate survival analysis of overall survival and disease-free survival in the 144 patients with osteosarcoma.

\begin{tabular}{|c|c|c|c|c|c|c|}
\hline \multirow[b]{2}{*}{ Variables } & \multicolumn{3}{|c|}{ Overall survival } & \multicolumn{3}{|c|}{ Disease-free survival } \\
\hline & $\mathrm{RR}$ & $95 \% \mathrm{CI}$ & P-value & $\mathrm{RR}$ & $95 \% \mathrm{CI}$ & P-value \\
\hline miR-26a expression & 5.724 & $1.008-10.941$ & $0.007^{\mathrm{a}}$ & 3.972 & $1.191-9.871$ & $0.014^{\mathrm{a}}$ \\
\hline Clinical stage & 2.541 & $1.157-4.214$ & $0.031^{\mathrm{a}}$ & 1.915 & $1.219-5.412$ & $0.041^{\mathrm{a}}$ \\
\hline Status of metastasis & 5.192 & $1.221-9.481$ & $0.011^{\mathrm{a}}$ & 4.021 & $1.092-10.327$ & $0.009^{\mathrm{a}}$ \\
\hline Response to chemotherapy & 2.198 & $1.871-6.517$ & $0.029^{\mathrm{a}}$ & 2.109 & $0.983-5.284$ & $0.037^{\mathrm{a}}$ \\
\hline
\end{tabular}

RR, relative risk; $95 \% \mathrm{CI}, 95 \%$ confidence interval; ${ }^{\mathrm{a}} \mathrm{P} \leq 0.05$.
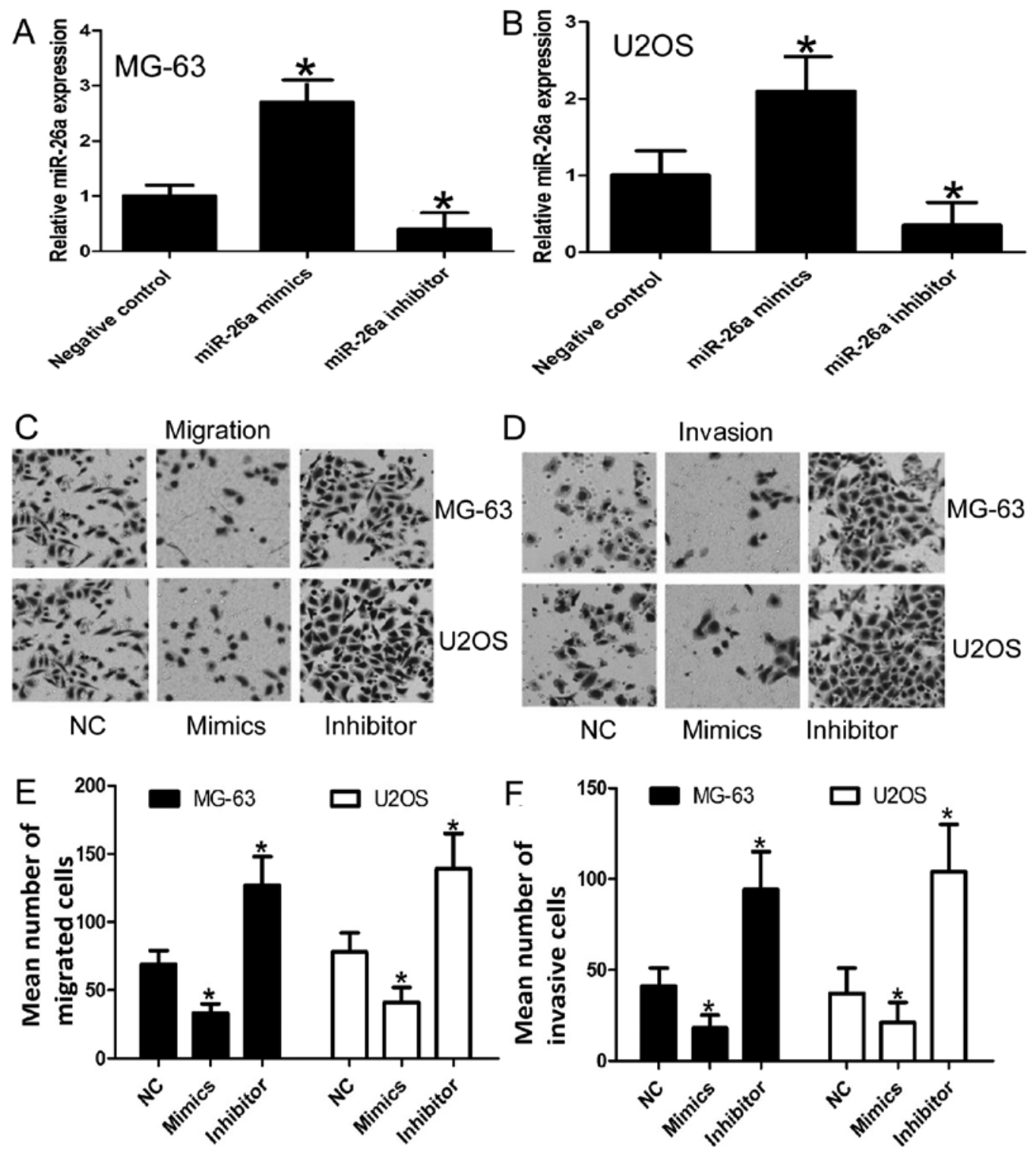

Figure 3. Relationship between miR-26a expression and the migration and invasion of osteosarcoma cell lines. (A and B) Transfection of MG-63 and U2OS cells with miR-26a mimics or an inhibitor increased and decreased, respectively, the expression of miR-26a as detected by qRT-PCR. (C-F) The effect of miR-26a on the migration and invasion of MG-63 and U2OS cells. Three independent experiments were performed in duplicate. Data are expressed as means \pm SD. The two-tailed Student's t-test was used to analyze significant differences; ${ }^{*} \mathrm{P}<0.05$.

further confirm EZH2 as a target gene for miR-26a, qRT-PCR and western blot analysis were used to detect the expression of EZH2 which was regulated by miR-26a in the MG-63 and U2OS cells. The expression of EZH2 was significantly downregulated after overexpression of miR-26a at the mRNA and protein levels in the osteosarcoma cells (Fig. 4B and C). 

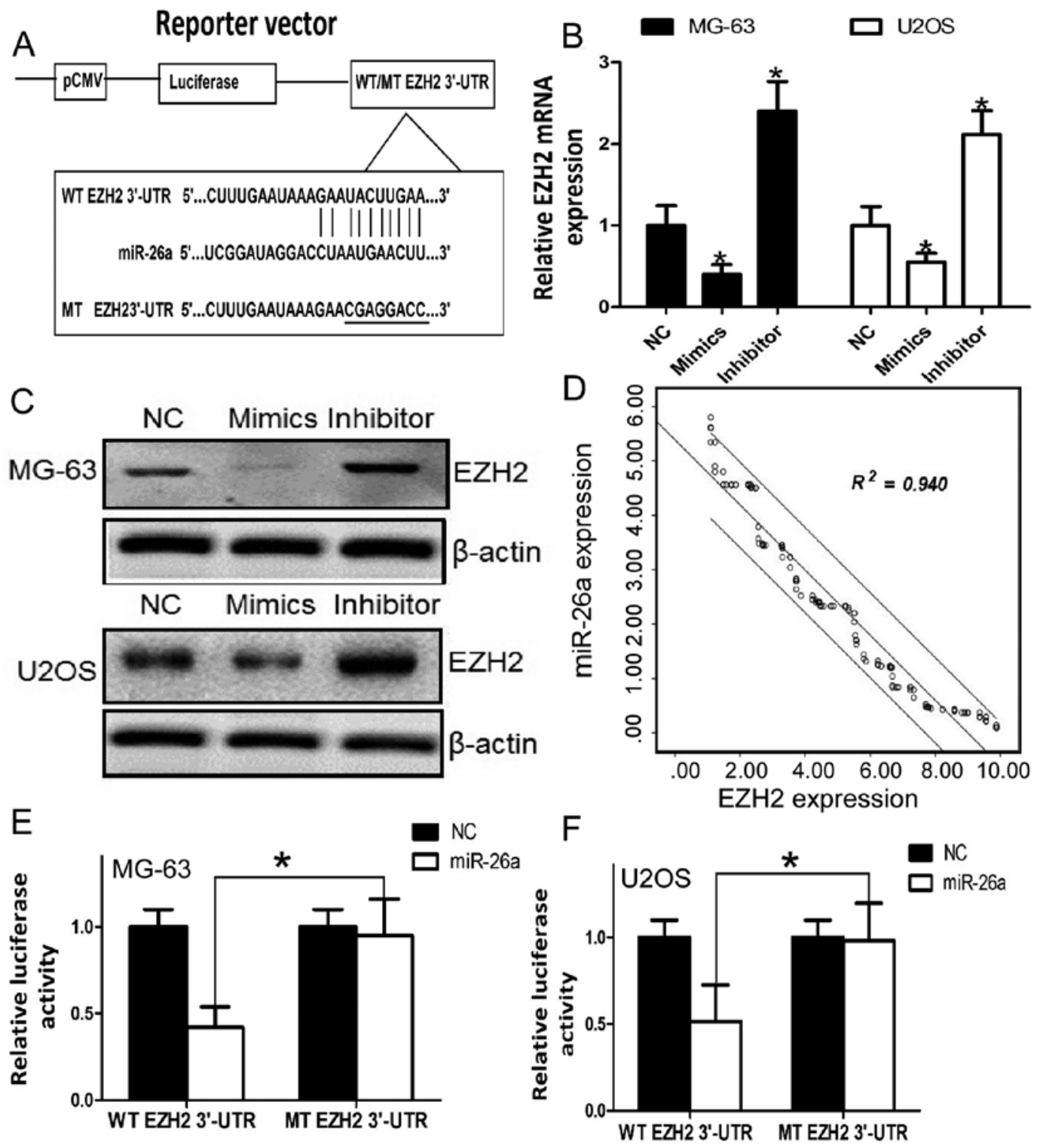

Figure 4.EZH2 3'-UTR is the direct target of miR-26a. (A) miR-26a and its putative binding sequence in the 3'-UTR of EZH2, and the diagram of the luciferase reporter plasmids with WT and MT EZH2 3'-UTR. (B and C) miR-26a inhibited the expression level of EZH2 at the mRNA and protein levels in the osteosarcoma cell lines. (D) The expression of miR-26a was negatively correlated with the expression of EZH2 in the osteosarcoma tissues. The relative luciferase activity in (E) MG-63 and (F) U2OS cells after the plasmid with WT or MT EZH2 3'-UTR was co-transfected with miR-26a mimics. Three independent experiments were performed in duplicate. Data are expressed as means \pm SD. Two-tailed Student's t-test was used to analyze the significant differences. "P<0.05. 3'-UTR, 3'-untranslated region; EZH2, enhancer of zeste homolog 2; WT, wild-type; MT, mutant.

Furthermore, we assessed the significance of the miR-26a and EZH2 correlation in osteosarcoma tissues. We determined the EZH2 mRNA and miR-26a expression in the same osteosarcoma specimens by qRT-PCR. As shown in Fig. 4D, a statistically significant inverse correlation was revealed by Spearman's correlation analysis between mRNA levels of miR-26a and EZH2 $(r=-0.969 ; \mathrm{P}<0.001)$. Taken together, our results suggest that miR-26a negatively regulates the expression of its target gene EZH2.

We further performed a luciferase reporter assay to verify whether miR-26a directly targets the 3'-UTR of EZH2 in osteosarcoma cells. The target sequence of EZH2 3'-UTR (WT 3'-UTR) or the mutant sequence (MT 3'-UTR) was cloned into a luciferase reporter vector (Fig. 4A). MG-63 and U2OS cells were then transfected with the WT or MT
3'-UTR vector and the miR-26a mimic. As shown in Fig. 4E, a significant decrease in luciferase activity was noted between the EZH2 WT 3'-UTR group and the negative control group $(\mathrm{P}<0.05)$. The repressive effect was abrogated by point mutation in the core binding sites of the EZH2 3'-UTR. A similar trend was also found in the U2OS cells (Fig. 4F). These results indicate that miR-26a exerts an inhibitory effect on EZH2 expression via interaction with the 3'-UTR of EZH2 in osteosarcoma cells.

\section{Discussion}

Metastasis is a major concern in the clinical treatment of osteosarcoma. The cure rate of osteosarcoma is $\sim 65 \%$ for localized osteosarcoma patients, whereas for patients presenting 
with metastases at the time of diagnosis, the survival rate is $25 \%(21,22)$. Several miRNAs have been reported to modulate tumor metastasis (23) including osteosarcoma (9). However, research concerning the aberrant expression and function of miRNAs in the progression and metastasis of osteosarcoma is still in its infancy.

In the present study, we demonstrated that miR-26a was weakly expressed in the osteosarcoma tissues when compared with that in the in non-cancerous bone tissues. The decreased expression of miR-26a in osteosarcoma tissues was also found to be significantly associated with adverse clinicopathological features. Moreover, we demonstrated that the miR-26a expression level was also predictive of disease progression and cancer-specific death. Our results showed that the patients with weak miR-26a expression had a significantly higher risk of cancer progression, cancer-specific death and shorter disease-free and overall survival time. Multivariate analysis demonstrated that low expression of miR-26a was a statistically significant risk factor affecting both disease-free survival and overall survival in osteosarcoma patients, which indicated that miR-26a expression may be an independent predictor of osteosarcoma progression and prognosis. The results provide initial evidence supporting miR-26a as a predictor of poor prognosis in osteosarcoma carcinoma.

To reveal the role of miR-26a in osteosarcoma cells, we assessed the effect of miR-26a on cell migration and invasion. Our results showed that miR-26a inhibited cell migration and invasion. Thus, miR-26a may be a novel tumor suppressor and may play an important role in the regulation of tumor metastasis of osteosarcoma. Next, we found the following evidence that miR-26a inhibits tumor metastasis in part by suppressing EZH2. (i) miRanda analysis showed that the 3'-UTR region of EZH2 mRNA contained the complementary sequence of miR-26a (Fig. 4A). (ii) Upregulation of miR-26a significantly reduced EZH2 expression at the mRNA and protein levels in osteosarcoma cells (Fig. 4B and C). (iii) The mRNA levels of miR-26a were inversely correlated with EZH2 levels in osteosarcoma tissues (Fig. 4D). (iv) Overexpression of miR-26a decreased the luciferase reporter activity of WT 3'-UTR but not MT 3'-UTR of EZH2 (Fig. 4E and F). These data support $\mathrm{EZH} 2$ as a downstream mediator of miR-26a function in osteosarcoma.

Downregulated miR-26a was reported to play an important role in the progression of tumor, and miR-26a functions as a potential tumor suppressor in several distinct cancer types $(5,10,13,14,24)$, but not including osteosarcoma. The underlying mechanisms responsible for the low expression of miR-26a in tumors have been investigated by several groups. Cellular homologue of avian myelocytomatosis virus oncogene (MYC) was reported to regulate miR-26a expression in thyroid anaplastic carcinoma and Burkitt's lymphoma (19,24,25). In addition, miR-26a was shown to be a tumor suppressor and may cause the inhibition of the precancerous molecule EZH2 in many types of cancer $(26,27)$. However the relationship between miR-26a and EZH2 in the tumorigenesis of human osteosarcoma has not been proven. Our study found that $\mathrm{EZH} 2$ is closely related to the expression of miR26a which may inhibit the metastasis of osteosarcoma cells. These results suggest that miR-26a regulates the process of metastasis and invasion by downregulating EZH2.
EZH2 which belongs to the family of polycomb group (PcG) proteins inhibits gene transcription through histone methylation (19) and plays a master regulatory role in many important cellular processes (28). Mounting evidence has shown that EZH2 is overexpressed in multiple cancer types and is associated with tumor invasive growth and aggressive clinical behavior, in prostate, renal, lung and breast cancer (29-32).

Moreover, EZH2 has been proven to enhance cell metastasis and neoplastic transformation (29,33-35). Consistent with our results, a previous study found that expression of EZH2 at the protein level was upregulated in osteosarcoma patient biopsy specimens when compare to normal bone, yet they also found that knockdown of EZH2 did not prevent osteosarcoma growth in vitro and in vivo (36). The discrepancies between this study and our research may be due to the different osteosarcoma cell lines selected by each study and the different focus on the functions of EZH2. In the previous study, the authors wanted to investigate the effect of EZH2 on osteosarcoma cell proliferation, while in our study, we aimed to prove that EZH2 was a downstream target of miR-26a. We did confirm that miR-26a affects the capacity of metastasis of osteosarcoma cells, thus EZH2 should have an effect on cell migration and invasion of osteosarcoma cells. Metastasis is the most important factor affecting the prognosis and progression of osteosarcoma. Thus, we suggest that overexpression of EZH2 in osteosarcoma holds significant promise for the advancement of cancer therapy, either in terms of improving diagnosis or predicting prognosis. Considering that EZH2 overexpression has been proven to be associated with poor prognosis in both metastatic breast and prostate cancer $(29,33)$ as well as in our research results, we believe that miR-26a plays an important role in the process of metastasis of osteosarcoma, and EZH2 is also involved in this process. The role of miR-26a and EZH2 in the proliferation of osteosarcoma warrants further detailed experiments with a higher number of samples.

In conclusion, our results demonstrate that miR-26a is downregulated in osteosarcoma, and reduced expression of miR-26a more frequently occurs in osteosarcoma tissues with adverse clinical stage and the presence of distant metastasis. Multivariate survival analyses demonstrated that loss of miR-26a is an independent prognostic factor for both diseasefree and overall survival in osteosarcoma. In addition, miR-26a inhibited the invasion and migration of osteosarcoma cells, and miR-26a directly inhibited EZH2 expression by targeting its 3'-UTR. Moreover, EZH2 was upregulated and inversely correlated with miR-26a in the osteosarcoma tissue samples. For the first time, the current data offer convincing evidence that the downregulation of miR-26a may be associated with tumor aggressiveness and tumor metastasis of osteosarcoma, and that miR-26a is an independent prognostic predictor for osteosarcoma patients. Our data suggest an important role for miR-26a in the molecular etiology of human osteosarcoma and implicate the potential application of miR-26a in the therapy of osteosarcoma.

\section{Acknowledgements}

The authors thank the local doctors and the patients who participated in the present study. 


\section{References}

1. Mirabello L, Troisi RJ and Savage SA: Osteosarcoma incidence and survival rates from 1973 to 2004: data from the Surveillance, Epidemiology, and End Results Program. Cancer 115: 1531-1543, 2009.

2. Teicher BA: Searching for molecular targets in sarcoma. Biochem Pharmacol 84: 1-10, 2012.

3. Marina N, Gebhardt M, Teot L and Gorlick R: Biology and therapeutic advances for pediatric osteosarcoma. Oncologist 9: 422-441, 2004

4. Gorlick R: Current concepts on the molecular biology of osteosarcoma. Cancer Treat Res 152: 467-478, 2009.

5. Calin GA and Croce CM: MicroRNA signatures in human cancers. Nat Rev Cancer 6: 857-866, 2006.

6. Kumar MS, Lu J, Mercer KL, Golub TR and Jacks T: Impaired microRNA processing enhances cellular transformation and tumorigenesis. Nat Genet 39: 673-677, 2007.

7. Tang M, Lin L, Cai H, Tang J and Zhou Z: MicroRNA-145 downregulation associates with advanced tumor progression and poor prognosis in patients suffering osteosarcoma. Onco Targets Ther 6: 833-838, 2013.

8. Shen L, Chen XD and Zhang YH: MicroRNA-128 promotes proliferation in osteosarcoma cells by downregulating PTEN. Tumour Biol: Oct 15, 2013 (Epub ahead of print).

9. Wang Y, Zhao W and Fu Q: miR-335 suppresses migration and invasion by targeting ROCK1 in osteosarcoma cells. Mol Cell Biochem 384: 105-111, 2013.

10. Kota J, Chivukula RR, O'Donnell KA, et al: Therapeutic microRNA delivery suppresses tumorigenesis in a murine liver cancer model. Cell 137: 1005-1017, 2009.

11. Ji J, Shi J, Budhu A, et al: MicroRNA expression, survival, and response to interferon in liver cancer. $\mathrm{N}$ Engl J Med 361: $1437-1447,2009$.

12. Ciarapica R, Russo G, Verginelli F, et al: Deregulated expression of miR-26a and Ezh2 in rhabdomyosarcoma. Cell Cycle 8: $172-175,2009$

13. Zhang B, Liu XX, He JR, et al: Pathologically decreased miR-26a antagonizes apoptosis and facilitates carcinogenesis by targeting MTDH and EZH2 in breast cancer. Carcinogenesis 32: 2-9, 2011.

14. Cabrera R and Szabo G: Another armed $\mathrm{CD} 4^{+} \mathrm{T}$ cell ready to battle hepatocellular carcinoma. Hepatology 58: 1-3, 2013.

15. Liu B, Wu X, Liu B, et al: MiR-26a enhances metastasis potential of lung cancer cells via AKT pathway by targeting PTEN. Biochim Biophys Acta 1822: 1692-1704, 2012.

16. Huse JT, Brennan C, Hambardzumyan D, et al: The PTENregulating microRNA miR-26a is amplified in high-grade glioma and facilitates gliomagenesis in vivo. Genes Dev 23: 1327-1337, 2009.

17. Zhang J, Han C and Wu T: MicroRNA-26a promotes cholangiocarcinoma growth by activating $\beta$-catenin. Gastroenterology 143: $246-256,2012$

18. Lu J, He ML, Wang L, et al: MiR-26a inhibits cell growth and tumorigenesis of nasopharyngeal carcinoma through repression of EZH2. Cancer Res 71: 225-233, 2011.

19. Sander S, Bullinger L, Klapproth K, et al: MYC stimulates $\mathrm{EZH} 2$ expression by repression of its negative regulator miR-26a Blood 112: 4202-4212, 2008.
20. Luzi E, Marini F, Sala SC, Tognarini I, Galli G and Brandi ML: Osteogenic differentiation of human adipose tissue-derived stem cells is modulated by the miR-26a targeting of the SMAD1 transcription factor. J Bone Miner Res 23: 287-295, 2008.

21. Gorlick R, Anderson P, Andrulis I, et al: Biology of childhood osteogenic sarcoma and potential targets for therapeutic development: meeting summary. Clin Cancer Res 9: 5442-5453, 2003.

22. Wittig JC, Bickels J, Priebat D, et al: Osteosarcoma: a multidisciplinary approach to diagnosis and treatment. Am Fam Physician 65: 1123-1132, 2002.

23. Sreekumar R, Sayan BS, Mirnezami AH and Sayan AE: MicroRNA control of invasion and metastasis pathways. Front Genet 2: 58, 2011.

24. Chang TC, Yu D, Lee YS, et al: Widespread microRNA repression by Myc contributes to tumorigenesis. Nat Genet 40: 43-50, 2008.

25. Dews M, Homayouni A, Yu D, et al: Augmentation of tumor angiogenesis by a Myc-activated microRNA cluster. Nat Genet 38: 1060-1065, 2006

26. Xu H, Yao Y, Smith LP and Nair V: MicroRNA-26a-mediated regulation of interleukin-2 expression in transformed avian lymphocyte lines. Cancer Cell Int 10: 15, 2010.

27. Dang X, Ma A, Yang L, et al: MicroRNA-26a regulates tumorigenic properties of $E Z H 2$ in human lung carcinoma cells. Cancer Genet 205: 113-123, 2012.

28. Sparmann A and van Lohuizen M: Polycomb silencers control cell fate, development and cancer. Nat Rev Cancer 6: 846-856, 2006.

29. Kleer CG, Cao Q, Varambally S, et al: EZH2 is a marker of aggressive breast cancer and promotes neoplastic transformation of breast epithelial cells. Proc Natl Acad Sci USA 100: 11606-11611, 2003.

30. Raaphorst FM, Meijer CJ, Fieret E, et al: Poorly differentiated breast carcinoma is associated with increased expression of the human polycomb group EZH2 gene. Neoplasia 5: 481-488, 2003.

31. $\mathrm{Xu} \mathrm{C}, \mathrm{Hou} Z$, Zhan $\mathrm{P}$, et al: $\mathrm{EZH} 2$ regulates cancer cell migration through repressing TIMP-3 in non-small cell lung cancer. Med Oncol 30: 713, 2013.

32. Wagener N, Macher-Goeppinger S, Pritsch M, et al: Enhancer of zeste homolog 2 (EZH2) expression is an independent prognostic factor in renal cell carcinoma. BMC Cancer 10: 524, 2010.

33. Varambally S, Dhanasekaran SM, Zhou M, et al: The polycomb group protein EZH2 is involved in progression of prostate cancer. Nature 419: 624-629, 2002.

34. Richter GH, Plehm S, Fasan A, et al: EZH2 is a mediator of EWS/FLI1 driven tumor growth and metastasis blocking endothelial and neuro-ectodermal differentiation. Proc Natl Acad Sci USA 106: 5324-5329, 2009

35. Croonquist PA and Van Ness B: The polycomb group protein enhancer of zeste homolog $2($ EZH 2$)$ is an oncogene that influences myeloma cell growth and the mutant ras phenotype. Oncogene 24: 6269-6280, 2005.

36. Sasaki H, Setoguchi T, Matsunoshita Y, Gao H, Hirotsu M and Komiya S: The knock-down of overexpressed EZH2 and BMI-1 does not prevent osteosarcoma growth. Oncol Rep 23: 677-684, 2010 . 